Communication

\title{
Enhancement of Crystallization Behaviors in Quaternary Composites Containing Biodegradable Polymer by Supramolecular Inclusion Complex
}

\author{
Li-Ting Lee ${ }^{1, *}$, Sheng-Ping $\mathrm{He}^{1}$ and Chih-Feng Huang ${ }^{2} \mathbb{D}$ \\ 1 Department of Materials Science and Engineering, Feng Chia University, Taichung 40724, Taiwan; \\ aaronhe0084@gmail.com \\ 2 Department of Chemical Engineering, i-Center for Advanced Science and Technology(iCAST), \\ National Chung Hsing University, Taichung 40227, Taiwan; HuangCF@dragon.nchu.edu.tw \\ * Correspondence: 1tlee@fcu.edu.tw; Tel.: +886-4-2451-7250 (ext. 5306)
}

Received: 24 November 2020; Accepted: 10 December 2020; Published: 12 December 2020

\begin{abstract}
Novel multi-component composites composed of the biodegradable polymer poly(ethylene adipate) (PEA), the water-soluble polymer poly(ethylene oxide) (PEO), poly(vinyl acetate) (PVAc), and a supramolecular-like inclusion complex (IC) made by $\alpha$-cyclodextrin $(\alpha-\mathrm{CD})$ and poly $(\varepsilon$-caprolactone) (PCL) (coded as PCL-CD-IC) are discussed in this work. The PCL-CD-IC was used to increase the crystallization rate of the miscible PEA/PEO/PVAc ternary blend that crystalized slower than neat PEA. Higher resolution SEM and TEM images displayed that PCL-CD-IC did not assemble notably in the quaternary composites. For the results of isothermal crystallization, the analysis of the Avrami equation demonstrated that the rate constant $k$ increased with the addition of PCL-CD-IC in the composites, suggesting that PCL-CD-IC provided more nucleation sites to promote the crystallization rate. The nucleation density increased with the addition of PCL-CD-IC, and the amount of spherulite also increased. Wide angle $\mathrm{X}$-ray results showed that the composites displayed similar diffraction patterns to neat PEA, meaning PEO, PVAc, and PCL-CD-IC would not change the crystal structures of PEA in the composites. The PCL-CD-IC, the supramolecular nucleation agent, demonstrated its superior ability to enhance the multi-component composites of biodegradable polymer in this study.
\end{abstract}

Keywords: crystallization and crystal; biodegradable polymers; supramolecular inclusion complex; quaternary polymer composites; $\alpha$-cyclodextrin

\section{Introduction}

Biodegradable polymers can be biodegraded to form simpler compounds and then redistributed through elemental cycles such as carbon and nitrogen [1-6]. Thus, they are more sustainable and environmentally friendly than traditional plastics. Poly(ethylene adipate) (PEA) is a biodegradable aliphatic polyester made from glycol and diacid. However, PEA shows a slower crystallization rate and poor thermal stability, which limits its application $[7,8]$. The crystalline properties of PEA can be enhanced by blending it with several nucleation agents $[7,8]$. It has been reported that graphene oxide (GO), as a nucleation agent, has good dispersion in a PEA/GO composite. Graphene oxide not only enhanced the nucleation density of PEA but also improved its spherulite growth rate [7]. Tang et al. [8] used a diamide derivative, $\mathrm{N}, \mathrm{N}^{\prime}$-ethylenebis(12-hydroxystearamide) (EBH), as a nucleation agent to promote the crystallization of PEA. It was found that the addition of EBH significantly reduced the crystallization time of PEA; moreover, the crystallization rate was accelerated. Addition of a nucleation agent is a useful way to modify the crystallization of PEA for its future applications.

Poly(ethylene oxide) (PEO) is a water-soluble polymer with low toxicity and good biocompatibility [9]. It can be used as a plasticizer to produce a plasticizing effect that can increase the chain mobility to 
enhance polymer crystallization. It has been reported by Eom et al. [10] that the addition of PEO can improve the ductility, hydrophilicity, and crystallinity of poly(L-lactic acid) (PLLA), and a PLLA-based film with PEO can be applied to flexible packaging materials. Poly(vinyl acetate) (PVAc) is fabricated by the free radical polymerization of vinyl acetate, which is biocompatible, non-toxic, environmentally friendly, and inexpensive [11]. The addition of PVAc can effectively improve the tensile strength, elongation at break, and Young's modulus of a polymeric system [11,12]. For example, the addition of PVAc to PEO/PLLA blends can increase the elongation at break of the blends [12]. Moreover, in the same system of PEO/PLLA blends, the compatibility between PEO and PLLA was improved with the presence of PVAc [12]. In the literature, the compatibility behaviors of PEA/PEO, PEA/PVAc, and PVAc/PEO have been discussed, and they all showed good compatibility [13-15]. Woo et al. [13] have discussed the compatibility and spherulite morphology of PEA/PEO blends. In thermal analysis results, a single glass transition temperature was presented for proving the good compatibility of the PEA/PEO blends. Lin et al. [14] studied the compatibility of PEA/PVAc blends. The PEA/PVAc blends were revealed showing a single homogeneous phase at the molten state, accompanied with a single glass transition temperature $\left(\mathrm{T}_{\mathrm{g}}\right)$ for each composition [14]. For the compatibility between PVAc and $\mathrm{PEO}$, relevant studies have been performed by Wu et al. [15], and they reported that the blending system of PVAc/PEO was compatible. According to the relevant information, it was shown that the blends PEA/PEO, PEA/PVAc, and PVAc/PEO were compatible.

Cyclodextrins (CDs) are products that can be extracted from the natural environment. In addition, they are also biocompatible, biodegradable, and safe for the environment. Generally, CDs can be divided into three types based on their chemical structures, which are called $\alpha-C D, \beta-C D$, and $\gamma-C D$. $\alpha$-Cyclodextrin, $\beta$-CD, and $\gamma$-CD have different chemical structures, showing 6,7 , and 8 glucopyranose units, respectively. They can also be used as potential additives to functional polymers such as biodegradable polymers [16]. Cyclodextrins can form inclusion complexes (ICs) with polymers, and ICs have supramolecular features in structure and function [17-19]. For example, $\alpha$-CD can form ICs with poly( $\varepsilon$-caprolactone) (PCL) and exhibit high $\alpha$-CD coverage on the PCL backbone [18]. It was reported that the IC made by $\alpha$-CD and PCL (PCL-CD-IC) may promote the crystallization behaviors of poly(butylene adipate) (PBA) with the effect of nucleation [19].

Supramolecular compounds, such as the stereocomplex of PLLA and poly(D-lactic acid) (PDLA) can be added to polymer-composite system as a nucleation agent to enhance the crystallization rate [20,21]. In this study, the novel PEA/PVAc/PEO/PCL-CD-IC quaternary composite was fabricated and discussed. The PCL-CD-IC was used as the supramolecular nucleation agent of the PEA/PVAc/PEO/PCL-CD-IC quaternary composite. Adding the PCL-CD-IC in the composite may increase the nucleation ability so that the crystallization behavior of the PEA/PVAc/PEO/PCL-CD-IC composites can be promoted. In this study, the composites with good compatibility when the relative weight ratio of PEA/PVAc/PEO $=80 / 10 / 10$ were selected as the typical samples for discovering the effect of adding PCL-CD-IC in the composites. The influence of the nucleation effect of PCL-CD-IC on the crystallization kinetics of the composites was the main subject discussed in this work.

\section{Materials and Methods}

\subsection{Materials and Preparation}

Poly(ethylene adipate) (PEA, $\mathrm{M}_{\mathrm{w}}=10,000 \mathrm{~g} / \mathrm{mol}$ ), poly(ethylene oxide) (PEO, $\left.\mathrm{M}_{\mathrm{w}}=200,000 \mathrm{~g} / \mathrm{mol}\right)$, poly( $\varepsilon$-caprolactone) (PCL, $\left.\mathrm{M}_{\mathrm{w}}=80,000 \mathrm{~g} / \mathrm{mol}\right)$, and $\alpha$-cyclodextrin $(\alpha-\mathrm{CD})$ were purchased from Sigma-Aldrich (Sigma-Aldrich, St. Louis, MO, USA). Poly(vinyl acetate) (PVAc, $\mathrm{M}_{\mathrm{w}}=184,000 \mathrm{~g} / \mathrm{mol}$ ) was purchased from Scientific Polymer Products (Scientific Polymer Products, New York, NY, USA). $\alpha$-Cyclodextrin was vacuum degassed and dried overnight at $80^{\circ} \mathrm{C}$ before using.

The preparation for the inclusion complex of PCL and $\alpha-C D$ (PCL-CD-IC) was using the solution mixing method. The PCL $(\sim 0.2 \mathrm{~g})$ and $\alpha-\mathrm{CD}(\sim 1.0 \mathrm{~g})$ were dissolved separately in acetone $(60 \mathrm{~mL})$ and distilled water $(10 \mathrm{~mL})$, respectively. After PCL and $\alpha$-CD were completely dissolved, PCL solution 
was added to the $\alpha$-CD solution and stirred vigorously at $60^{\circ} \mathrm{C}$ for $3 \mathrm{~h}$ for the complexation process. The entire complexation process was carried out by further stirring the solution overnight at room temperature, and PCL-CD-IC was obtained as the precipitate in the solution. After filtrating out the PCL-CD-IC from the solution, the PCL-CD-IC was also washed with acetone and distilled water several times to remove the uncomplexed PCL and $\alpha-C D$, respectively. The PCL-CD-IC after washing away the uncomplexed PCL and $\alpha-C D$ was vacuum dried at $45^{\circ} \mathrm{C}$ for one week before using. A similar method to prepare PCL-CD-IC has also been reported in the literature [18].

The quaternary PEA/PEO/PVAc/PCL-CD-IC composites were fabricated by the solution blending method. To prepare a PEA/PEO/PVAc/PCL-CD-IC solution, PCL-CD-IC was first dispersed in chloroform by ultrasonic treatment $(20 \mathrm{~min})$ to make the PCL-CD-IC solution, and then a certain amount of it was added to a PEA/PEO/PVAc solution (with chloroform as the solvent), continuously stirring for $10 \mathrm{~min}$. It should be noted that the concentration of the PEA/PEO/PVAc/PCL-CD-IC solutions was approximately $3 \mathrm{wt} \%$. For the content of PEA, PEO, PVAc, and PCL-CD-IC in the solutions, it was presented by their relative weight ratio, for example, PEA/PEO/PVAc/PCL-CD-IC = 80/10/10/0.5. The PEA/PEO/PVAc/PCL-CD-IC solutions were casted by evaporating the solvent at $40^{\circ} \mathrm{C}$ and vacuum drying for 2 days at $45^{\circ} \mathrm{C}$. The obtained specimens were used for the following characterizations and measurements.

\subsection{Investigations on Thermal and Crystallization Behaviors}

We studied the thermal properties and crystallization behaviors with a differential scanning calorimeter (DSC, PerkinElmer DSC-8500, PerkinElmer, Waltham, MA, USA). In order to detect the general thermal behaviors of the specimens, a scan rate of $20^{\circ} \mathrm{C} / \mathrm{min}$ was used. Regarding the isothermal crystallization treatment of PEA/PEO/PVAc/PCL-CD-IC composites, the specimens were first heated and kept at $100{ }^{\circ} \mathrm{C}$ for 3 min to erase thermal history, and then rapidly cooled to various crystallization temperatures $\left(\mathrm{T}_{\mathrm{C}}=16,18,20,22^{\circ} \mathrm{C}\right)$ to complete crystallization. Relevant information about isothermal crystallization was recorded for further analysis.

\subsection{Structural Identification with ${ }^{1} H$ Nuclear Magnetic Resonance $\left({ }^{1} H\right.$ NMR) Spectra}

To identify the structure of PCL-CD-IC, the ${ }^{1} \mathrm{H}$ nuclear magnetic resonance $\left({ }^{1} \mathrm{H}\right.$ NMR) spectra were measured by the Varian Unity Inova 400 (Agilent, Santa Clara, CA, USA). The solvent of DMSO- $\mathrm{d}_{6}$ was selected to dissolve the specimens. The signal of DMSO- $\mathrm{d}_{6}$ at $\delta=2.5 \mathrm{ppm}$ was used as the internal standard for our characterization.

\subsection{Studies of Fourier-Transform Infrared Spectroscopy (FTIR) Spectra}

Fourier-transform infrared spectroscopy (FTIR) spectra of the composites were recorded with the PerkinElmer Frontier $^{\mathrm{TM}}$ (Perkin Elmer, Waltham, MA, USA) at wavenumbers from 400 to $4000 \mathrm{~cm}^{-1}$. The samples for FTIR measurements were prepared on $\mathrm{KBr}$ disks using the solution-casting method. The resolution of each measurement was $2 \mathrm{~cm}^{-1}$.

\subsection{Discussions on Crystalline Structures with Wide-Angle X-ray Diffraction (WAXD)}

We employed wide-angle X-ray diffraction (WAXD) to discuss the crystalline structures of PEA/PEO/PVAc/PCL-CD-IC composites. A Bruker D2 PHASER diffractometer (Bruker, Billerica, MA, USA) with $\mathrm{Cu}-\mathrm{K} \alpha$ radiation $(\lambda=0.154 \mathrm{~nm})$ was applied. The samples used for the WAXD studies were crystalized isothermally at $22{ }^{\circ} \mathrm{C}$ before the measurements. The WAXD experiments were performed at a scan rate of $1^{\circ} / \mathrm{min}$ between $2 \theta=5^{\circ}$ and $50^{\circ}$.

\subsection{Morphological Observation}

Scanning electron microscopy (SEM, Hitachi S3000, Hitachi, Tokyo, Japan) and transmission electron microscopy (TEM, JEM-1400, JEOL, Tokyo, Japan) were used to study the dispersion and 
morphology of the PCL-CD-ICs in composites. The samples for SEM were coated with gold so that the conductivity could be increased. The samples for TEM were prepared on copper grids by solution casting. The spherulite morphology of PEA in the composites was also discussed and observed through a polarization optical microscope (POM, Olympus CX41, Olympus, Tokyo, Japan) equipped with a Linkam THMS-600 hot stage. Prior to POM observation, the specimens were melted at $100{ }^{\circ} \mathrm{C}$ for $3 \mathrm{~min}$ and then crystalized isothermally at $30^{\circ} \mathrm{C}$.

\section{Results}

Figure 1 shows the DSC thermograms of neat PCL, $\alpha-C D$, and PCL-CD-IC. Obviously, for PCL-CD-IC, the melting transition of PCL was diminished. This result indicates that the crystallization of PCL was affected because of the formation of inclusion complexes between PCL and $\alpha-C D$. The crystallization of PCL was restricted because most of the PCL chains were covered by $\alpha-C D$ during the formation of the PCL-CD-IC.

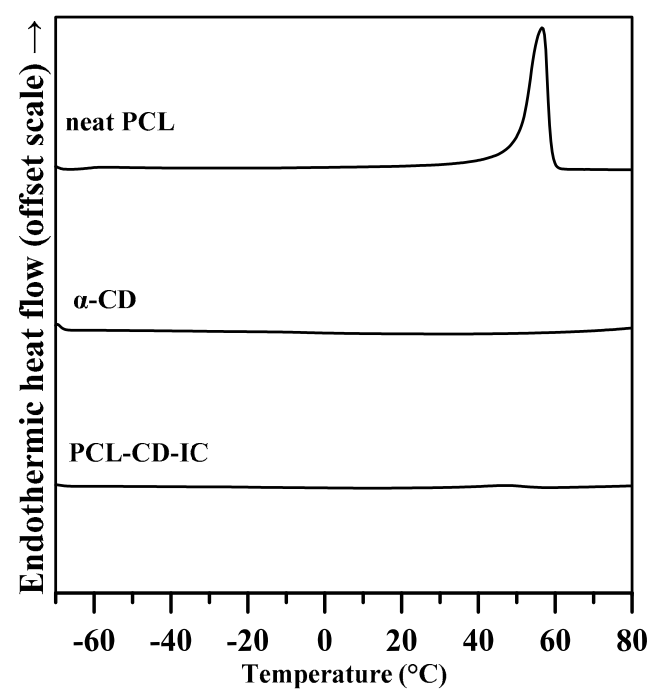

Figure 1. Differential scanning calorimeter (DSC) thermograms of neat poly( $\varepsilon$-caprolactone) (PCL), $\alpha$-cyclodextrin $(\alpha-C D)$, and a PCL-CD-inclusion complex (IC).

The stoichiometry of PCL-CD-IC was estimated from the ${ }^{1} \mathrm{H}$ NMR spectra shown in Supplementary Materials Figure S1. According to the spectra in Figure S1, the molar ratio of PCL repeating unit to $\alpha-C D$ and the coverage ratio of PCL-CD-IC can be related to the ratio of the integral intensity of $4.8 \mathrm{ppm}$ $(\mathrm{CH}$ of $\alpha-\mathrm{CD})$ to the integral intensity of $1.5 \mathrm{ppm}(\mathrm{CH}$ of $\mathrm{PCL})$. Similar identifications and estimations have also been mentioned in the literature [22,23]. The molar ratio of the PCL repeating unit to $\alpha-C D$ was estimated to be 1.15 , and the coverage ratio of PCL-CD-IC was approximately $76 \%$. The related results show that most of the PCL chains in the PCL-CD-IC were covered by $\alpha$-CD. The FTIR spectra of neat PCL, $\alpha-C D$, and PCL-CD-IC are demonstrated in Figure S2 in the Supplementary Materials. We found that the hydroxyl-stretching band of $\alpha-\mathrm{CD}\left(3367 \mathrm{~cm}^{-1}\right)$ was different with that of PCL-CD-IC $\left(3391 \mathrm{~cm}^{-1}\right)$. In addition, the carbonyl-stretching band of PCL $\left(1725 \mathrm{~cm}^{-1}\right)$ was different than that of PCL-CD-IC $\left(1739 \mathrm{~cm}^{-1}\right)$. The shifts of the hydroxyl-stretching band and the carbonyl-stretching band revealed by FTIR spectra further indicate the presence of inclusion between PCL and $\alpha-C D$ and the interactions between the hydroxyl groups of $\alpha-C D$ and the carbonyl groups of PCL in the PCL-CD-IC. Similar spectral features have also been shown to characterize the occurrence of inclusion in the literature [24,25].

The miscibility of the PEA/PEO/PVAc/PCL-CD-IC quaternary composite was confirmed by a DSC thermal scan, and the typical result of the PEA/PEO/PVAc/PCL-CD-IC $=80 / 10 / 10 / 1$ composite is shown in Figure S3 in the Supplementary Materials. As shown in Figure S3, a single glass transition temperature $\left(\mathrm{T}_{\mathrm{g}}\right)$ was found for the PEA/PEO/PVAc/PCL-CD-IC composite, demonstrating the miscibility of the 
composite. The isothermal crystallization kinetics of the PEA/PEO/PVAc/PCL-CD-IC quaternary composites were thoroughly investigated in this study. Figure 2 displays the DSC measurements of neat PEA and the PEA/PEO/PVAc/PCL-CD-IC quaternary composites at different crystallization temperatures $\left(\mathrm{T}_{\mathrm{c}}{ }^{\prime}\right)$. The typical results of PEA/PEO/PVAc/PCL-CD-IC $=80 / 10 / 10 / x$ composites are presented herein. It should be noted that the " $x$ " values were $0,0.5,1$, and 2 , which represent the relative weight ratio of PCL-CD-IC in the composite. Firstly, it was found that for PEA/PEO/PVAc/PCL-CD-IC = 80/10/10/0 specimen without adding PCL-CD-IC, the crystallization peak moved to a longer crystallization time. This result means that the presence of PEO and PVAc in the composite would slow down the isothermal crystallization of PEA. On the other hand, adding PCL-CD-IC can further promote the isothermal crystallization of PEA in the composites. As shown in Figure 2a-d, we found that under the same $T_{c}$, the crystallization time of PEA/PEO/PVAc/PCL-CD-IC $=80 / 10 / 10 / x$ composites decreased noticeably with the increase in PCL-CD-IC content. The addition of PCL-CD-IC effectively enhanced the isothermal crystallization of PEA in the composites. The isothermal crystallization kinetics of the composites can be described by the Avrami equation [26,27]. The analysis method using the Avrami equation is described in detail in the Supplementary Materials. Figure 3 demonstrates the Avrami plots of neat PEA and the PEA/PEO/PVAc/PCL-CD-IC quaternary composites. It is clear that all curves in the Avrami plots show good linearity. The evaluated values obtained through the analysis are presented in Table 1. We found that the $n$ values of neat PEA and PEA/PEO/PVAc/PCL-CD-IC quaternary composites were similar and between 2 and 3, which implies that the crystallization mechanism of PEA would not be significantly influenced with the addition of PEO, PVAc, and PCL-CD-IC. The values of rate constant $\mathrm{k}$ and $1 / \mathrm{t}_{0.5}$ tended to increase with an increase in the PCL-CD-IC content in the composites, indicating that PCL-CD-IC increased the crystallization rate of the composites. The PCL-CD-IC can be an effective nucleation agent for the crystallization of PEA in the quaternary PEA/PEO/PVAc/PCL-CD-IC composites.
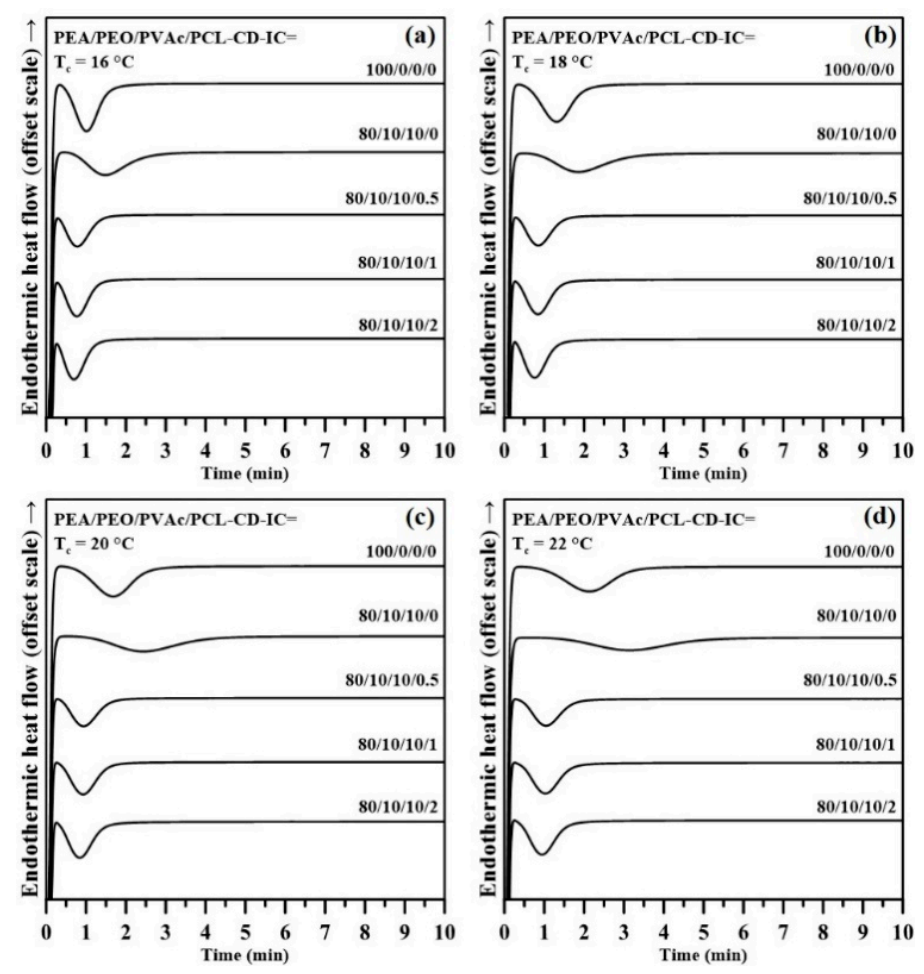

Figure 2. DSC measurements of neat poly(ethylene adipate) (PEA) and PEA/poly(ethylene oxide) (PEO)/poly(vinyl acetate) (PVAc)/PCL-CD-IC $=80 / 10 / 10 / x$ composites at $(\mathbf{a}) \mathrm{T}_{\mathrm{C}}=16{ }^{\circ} \mathrm{C},(\mathbf{b}) \mathrm{T}_{\mathrm{C}}=18{ }^{\circ} \mathrm{C}$, (c) $\mathrm{T}_{\mathrm{C}}=20^{\circ} \mathrm{C}$, and (d) $\mathrm{T}_{\mathrm{C}}=22^{\circ} \mathrm{C}$. The $\mathrm{x}$ values are the relative weight ratios of the PCL-CD-ICs in the composites, which were $0,0.5,1$, and 2 , respectively. 

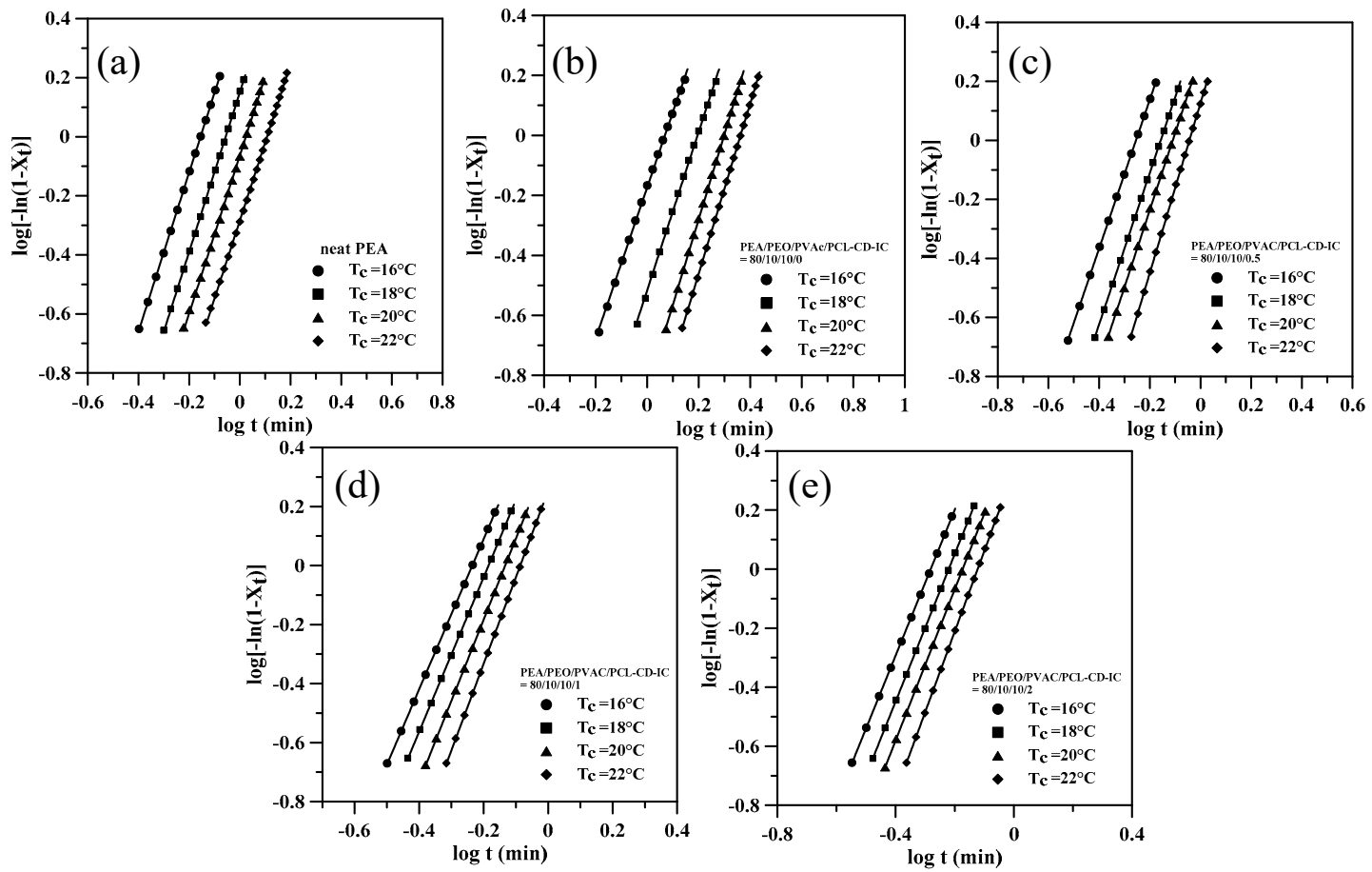

Figure 3. The Avrami plots derived from DSC measurements of (a) neat PEA, (b) PEA/PEO/PVAc/PCL-CD-IC = 80/10/10/0, (c) PEA/PEO/PVAc/PCL-CD-IC = 80/10/10/0.5, (d) PEA/PEO/PVAc/PCL-CD-IC = 80/10/10/1, and (e) PEA/PEO/PVAc/PCL-CD-IC = 80/10/10/2 composite.

Table 1. The Avrami parameters derived from DSC measurements for neat PEA and $\mathrm{PEA} / \mathrm{PEO} / \mathrm{PVAc} / \mathrm{PCL}-\mathrm{CD}-\mathrm{IC}=80 / 10 / 10 / \mathrm{x}$ composites. The $\mathrm{x}$ values are the relative weight ratios of PCL-CD-ICs in the composites and were $0,0.5,1$ and 2.

\begin{tabular}{|c|c|c|c|c|c|}
\hline $\begin{array}{l}\text { PEA/PEO/PVAc/PCL-CD-IC } \\
\text { (Relative Weight Ratio) }\end{array}$ & $\mathrm{T}_{\mathrm{c}}\left({ }^{\circ} \mathrm{C}\right)$ & $n$ & $k\left(\min ^{-n}\right)$ & $t_{0.5}(\min )$ & $1 / t_{0.5}\left(\min ^{-1}\right)$ \\
\hline \multirow[t]{4}{*}{$100 / 0 / 0 / 0$} & 16 & 2.70 & 2.62 & 0.61 & 1.64 \\
\hline & 18 & 2.70 & 1.42 & 0.77 & 1.30 \\
\hline & 20 & 2.68 & 0.87 & 0.92 & 1.09 \\
\hline & 22 & 2.65 & 0.52 & 1.11 & 0.90 \\
\hline \multirow[t]{4}{*}{$80 / 10 / 10 / 0$} & 16 & 2.52 & 0.67 & 1.01 & 0.99 \\
\hline & 18 & 2.64 & 0.31 & 1.37 & 0.73 \\
\hline & 20 & 2.84 & 0.14 & 1.75 & 0.57 \\
\hline & 22 & 2.84 & 0.09 & 2.03 & 0.49 \\
\hline \multirow[t]{4}{*}{$80 / 10 / 10 / 0.5$} & 16 & 2.52 & 3.48 & 0.53 & 1.90 \\
\hline & 18 & 2.57 & 2.53 & 0.60 & 1.66 \\
\hline & 20 & 2.61 & 1.93 & 0.68 & 1.48 \\
\hline & 22 & 2.88 & 1.34 & 0.80 & 1.26 \\
\hline \multirow[t]{4}{*}{$80 / 10 / 10 / 1$} & 16 & 2.55 & 3.98 & 0.50 & 1.99 \\
\hline & 18 & 2.62 & 3.05 & 0.57 & 1.76 \\
\hline & 20 & 2.75 & 2.33 & 0.64 & 1.55 \\
\hline & 22 & 2.92 & 1.80 & 0.72 & 1.39 \\
\hline \multirow[t]{4}{*}{$80 / 10 / 10 / 2$} & 16 & 2.54 & 5.82 & 0.43 & 2.31 \\
\hline & 18 & 2.50 & 3.55 & 0.52 & 1.92 \\
\hline & 20 & 2.56 & 2.78 & 0.58 & 1.72 \\
\hline & 22 & 2.73 & 2.16 & 0.66 & 1.52 \\
\hline
\end{tabular}

The typical SEM and TEM images of the composite are demonstrated in Figure 4a,b. The images were acquired from the measurements of the PEA/PEO/PVAc/PCL-CD-IC $=80 / 10 / 10 / 1$ composite, with SEM and TEM magnifications of 2000× and 25,000×, respectively. As shown in the SEM image of Figure 4a, we found that the PCL-CD-IC was well dispersed in the composite, and there was no 
obvious agglomeration between each other, showing particle sizes of approximately a few micrometers. In addition, as shown in Figure 4b, the PCL-CD-IC can also be observed by TEM, and a particle size of approximately $3-5 \mu \mathrm{m}$ at the submicron scale was confirmed.

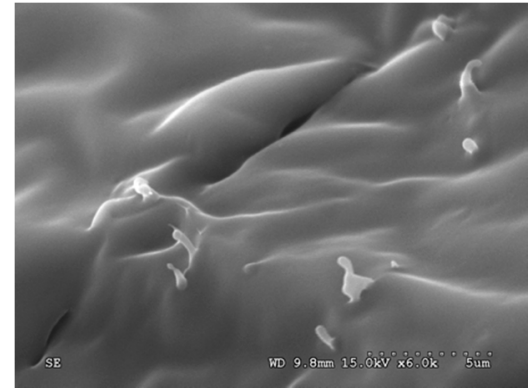

(a)

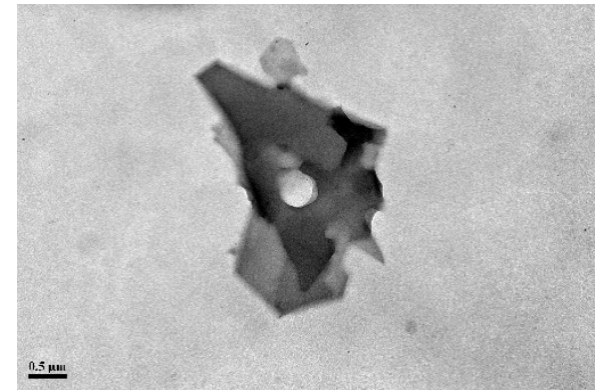

(b)

Figure 4. (a) SEM and (b) TEM images of the PEA/PEO/PVAc/PCL-CD-IC = 80/10/10/1 composite.

Figure 5 shows the POM morphologies of neat PEA and quaternary PEA/PEO/PVAc/PCL-CD-IC composites with different quaternary compositions. All samples were crystalized at $30^{\circ} \mathrm{C}$. It was found that the addition of PCL-CD-IC increased the number of spherulites of PEA in the composites. With the presence of PCL-CD-IC, the composite can obtain more nucleation sites, and the nucleation density can also be increased. The PCL-CD-IC is an effective nucleation agent to promote crystallization in PEA/PEO/PVAc/PCL-CD-IC composites.
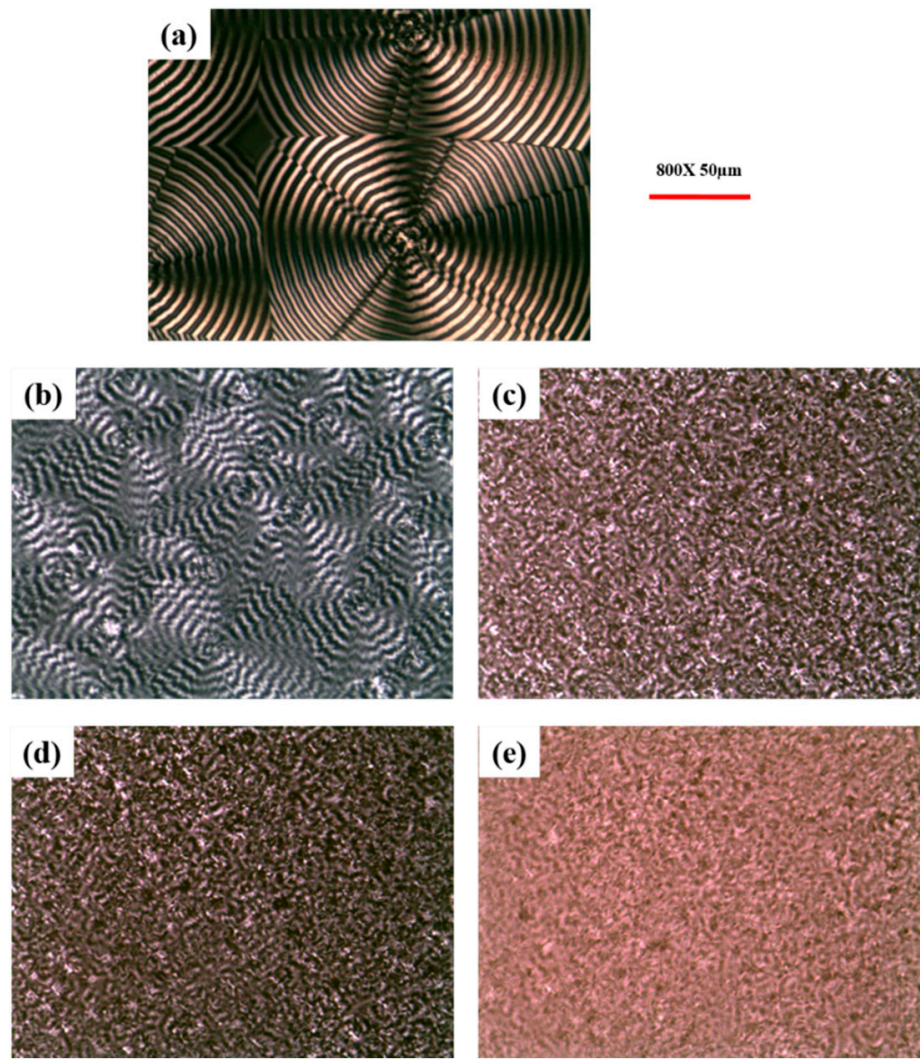

Figure 5. The polarization optical microscope (POM) graphs of (a) neat PEA, (b) $\mathrm{PEA} / \mathrm{PEO} / \mathrm{PVAc} / \mathrm{PCL}-\mathrm{CD}-\mathrm{IC}=80 / 10 / 10 / 0, \quad$ (c) $\mathrm{PEA} / \mathrm{PEO} / \mathrm{PVAc} / \mathrm{PCL}-\mathrm{CD}-\mathrm{IC}=80 / 10 / 10 / 0.5$, (d) $\mathrm{PEA} / \mathrm{PEO} / \mathrm{PVAc} / \mathrm{PCL}-\mathrm{CD}-\mathrm{IC}=80 / 10 / 10 / 1$, and $(\mathbf{e}) \mathrm{PEA} / \mathrm{PEO} / \mathrm{PVAc} / \mathrm{PCL}-\mathrm{CD}-\mathrm{IC}=80 / 10 / 10 / 2$ composite. All samples were crystalized at $30^{\circ} \mathrm{C}$. 
Figure 6 presents the WAXD results of neat PEA and PEA/PEO/PVAc/PCL-CD-IC composites. The quaternary compositions of the PEA/PEO/PVAc/PCL-CD-IC composites shown here were 80/10/10/0 and 80/10/10/1. Due to the reflection from the (111), (110), and (020) crystalline planes [28-30], neat PEA presented three main diffraction peaks at $2 \theta=20.4^{\circ}, 22.3^{\circ}$, and $24.7^{\circ}$, respectively. We found that PEA had no peak shift after forming the composite. The WAXD patterns of PEA were very similar to those of the PEA/PEO/PVAc/PCL-CD-IC composites, which means that PEA will not change its crystalline structures after adding of PEO, PVAc, and PCL-CD-IC. In addition, since the X-ray reflections of the quaternary composite seem to be less pronounced, the crystallinity of the quaternary composite should be slightly lower.

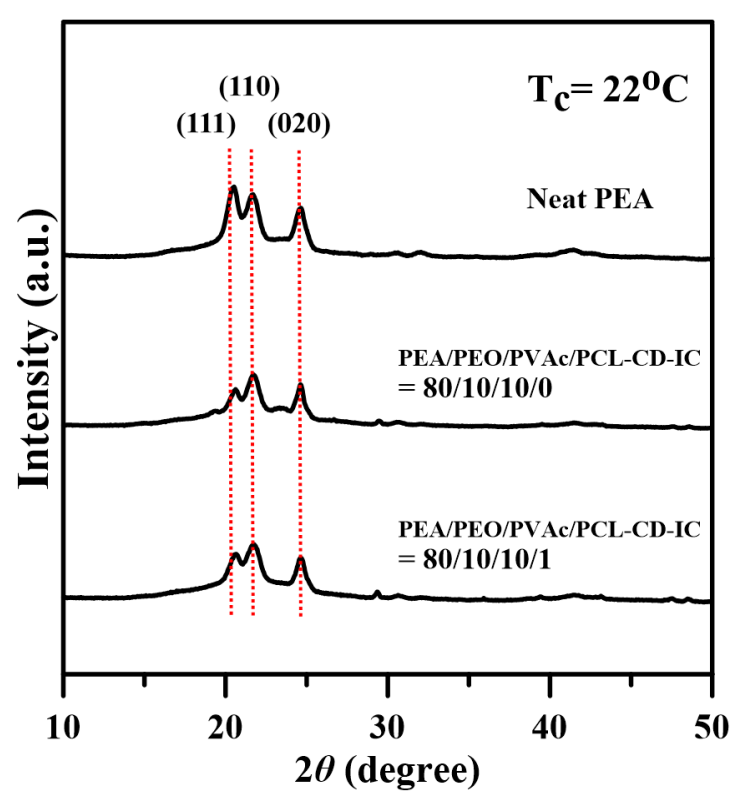

Figure 6. The wide-angle $X$-ray diffraction (WAXD) results of neat PEA and PEA/PEO/PVAc/PCL-CD-IC composites crystalized at $22{ }^{\circ} \mathrm{C}$. The quaternary compositions of the PEA/PEO/PVAc/PCL-CD-IC composites shown here were 80/10/10/0 and 80/10/10/1.

\section{Conclusions}

The novel quaternary biodegradable polymer composites of PEA/PEO/PVAc/PCL-CD-IC were investigated in this study. In studies of quaternary biodegradable polymer composites, this work was the first to use supramolecular inclusion complexes in enhancing the crystallization behaviors. An inclusion complex of PCL-CD-IC was successfully prepared and studied by DSC, FTIR, and ${ }^{1} \mathrm{H}$ NMR. The PCL-CD-IC was further used to promote the crystallization rate of quaternary PEA/PEO/PVAc/PCL-CD-IC composites. We found that PCL-CD-IC can be well-dispersed in the quaternary composites of PEA/PEO/PVAc/PCL-CD-IC. In the analyses of isothermal crystallization by the Avrami equation, the rate constant $\mathrm{k}$ was increased when the content of PCL-CD-IC was increased. By this result, it can be indicated that PCL-CD-IC promoted the crystallization rate of PEA in the composite. According to the WAXD results, the quaternary composites of PEA/PEO/PVAc/PCL-CD-IC displayed similar diffraction patterns to neat PEA. The POM observations revealed that the nucleation density of the quaternary composites can be significantly increased with the presence of PCL-CD-IC. The PCL-CD-IC with a supramolecular structure can effectively promote the crystallization of the novel biodegradable polymer composites composed of PEA, PEO, PVAc, and PCL-CD-IC. The composites investigated in this study may have the potential to be applied in biodegradable plastics and materials for biomedical and agricultural end uses.

Supplementary Materials: The following are available online at http://www.mdpi.com/2073-4352/10/12/1137/s1, Figure S1: ${ }^{1} \mathrm{H}$ NMR spectra of $\alpha-\mathrm{CD}$, neat PCL, and PCL-CD-IC; Figure S2: FTIR spectra of neat PCL, $\alpha-C D$, 
and PCL-CD-IC; Figure S3: DSC thermal scan of the PEA/PEO/PVAc/PCL-CD-IC = 80/10/10/1 composite; The analysis method of isothermal crystallization using the Avrami equation; References of Supplementary Materials.

Author Contributions: L.-T.L. was responsible for developing the research outline, designing the experiments, and writing most of the paper. S.-P.H. was responsible for performing the experiments and data analysis of thermal and microscopy results. C.-F.H. was responsible for ${ }^{1} \mathrm{H}$ NMR experiments and analysis. All authors have read and agreed to the published version of the manuscript.

Funding: This research was funded by the Ministry of Science and Technology, Taiwan, grant number MOST 108-2221-E-035-062 and MOST 109-2221-E-035-079.

Acknowledgments: The authors appreciate the financial support from the Ministry of Science and Technology, Taiwan (MOST 108-2221-E-035-062 and MOST 109-2221-E-035-079).

Conflicts of Interest: The authors declare no conflict of interest.

\section{References}

1. Chandra, R.; Rustgi, R. Biodegradable polymers. Prog. Polym. Sci. 1998, 23, 1273-1335. [CrossRef]

2. Vroman, I.; Tighzert, L. Biodegradable polymers. Materials 2009, 2, 307-344. [CrossRef]

3. Gross, R.A.; Kalra, B. Biodegradable polymers for the environment. Science 2002, 297, 803-807. [CrossRef] [PubMed]

4. Safari, S.; van de Ven, T.G. Effect of crystallization conditions on the physical properties of a two-layer glassine paper/polyhydroxybutyrate structure. J. Mater. Sci. 2015, 50, 3686-3696. [CrossRef]

5. Chaiwutthinan, P.; Chuayjuljit, S.; Srasomsub, S.; Boonmahitthisud, A. Composites of poly(lactic acid)/poly(butylene adipate-co-terephthalate) blend with wood fiber and wollastonite: Physical properties, morphology, and biodegradability. J. Appl. Polym. Sci. 2019, 136. [CrossRef]

6. Luckachan, G.E.; Pillai, C.K.S. Biodegradable polymers-a review on recent trends and emerging perspectives. J. Polym. Environ. 2011, 19, 637-676. [CrossRef]

7. Jiang, Z.; Qiu, Z. Unusual crystallization behavior of biodegradable poly(ethylene adipate) based nanocomposites induced by graphene oxide. RSC Adv. 2015, 5, 55486-55491. [CrossRef]

8. Tang, J.; Li, L.; Wang, X.; Yang, J.; Liang, X.; Li, Y.; Ma, H.; Zhou, S.; Wang, J. Tailored crystallization behavior, thermal stability, and biodegradability of poly(ethylene adipate): Effects of a biocompatible diamide nucleating agent. Polym. Test 2020, 81. [CrossRef]

9. Hsieh, Y.T.; Nurkhamidah, S.; Woo, E.M. Lamellar orientation and interlamellar cracks in co-crystallized poly(ethylene oxide)/poly(L-lactic acid) blend. Polym. J. 2011, 43, 762-769. [CrossRef]

10. Eom, Y.; Choi, B.; Park, S.-I. A study on mechanical and thermal properties of PLA/PEO blends. J. Polym. Environ. 2019, 27, 256-262. [CrossRef]

11. Abdelghany, A.; Meikhail, M.; Asker, N. Synthesis and structural-biological correlation of PVC/PVAc polymer blends. J. Mater. Res. Technol-JMRT 2019, 8, 3908-3916. [CrossRef]

12. Kim, K.S.; Chin, I.J.; Yoon, J.S.; Choi, H.J.; Lee, D.C.; Lee, K.H. Crystallization behavior and mechanical properties of poly(ethylene oxide)/poly(L-lactide)/poly(vinyl acetate) blends. J. Appl. Polym. Sci. 2001, 82, 3618-3626. [CrossRef]

13. Lin, J.H.; Woo, E.M. Correlation between interactions, miscibility, and spherulite growth in crystalline/crystalline blends of poly(ethylene oxide) and polyesters. Polymer 2006, 47, 6826-6835. [CrossRef]

14. Chang, C.S.; Woo, E.M.; Lin, J.H. Miscibility with asymmetrical interactions in blends of two carbonyl-containing polymers: Poly(vinyl acetate) with aliphatic polyesters. Macromol. Chem. Phys. 2006, 207, 1404-1413. [CrossRef]

15. Wu, W.; Chiu, W.; Liau, W. Casting solvent effect on crystallization behavior of poly(vinyl acetate)/poly(ethylene oxide) blends: DSC study. J. Appl. Polym. Sci. 1997, 64, 411-421. [CrossRef]

16. Szente, L.; Fenyvesi, É. Cyclodextrin-enabled polymer composites for packaging. Molecules 2018, $23,1556$. [CrossRef]

17. Folch-Cano, C.; Yazdani-Pedram, M.; Olea-Azar, C. Inclusion and functionalization of polymers with cyclodextrins: Current applications and future prospects. Molecules 2014, 19, 4066. [CrossRef]

18. Dong, T.; Mori, T.; Pan, P.; Kai, W.; Zhu, B.; Inoue, Y. Crystallization behavior and mechanical properties of poly(E-caprolactone)/cyclodextrin biodegradable composites. J. Appl. Polym. Sci. 2009, 112, 2351-2357. [CrossRef] 
19. Prasannan, A.; Bich-Tram, T.-L.; Hsu, D.-Y.; Hong, P.-D.; Pan, G.-R. Nucleation effects of $\alpha$-cyclodextrin inclusion complexes on the crystallization behavior of biodegradable poly(1,4-butylene adipate). Crystengcomm. 2013, 15, 5119-5126. [CrossRef]

20. Chang, L.; Woo, E.M. Crystallization of poly(3-hydroxybutyrate) with stereocomplexed polylactide as biodegradable nucleation agent. Polym. Eng. Sci. 2012, 52, 1413-1419. [CrossRef]

21. Chen, Y.A.; Wu, T.M. Crystallization kinetics of poly(1,4-butylene adipate) with stereocomplexed poly(lactic acid) serving as a nucleation agent. Ind. Eng. Chem. Res. 2014, 53, 16689-16695. [CrossRef]

22. Harada, A.; Kawaguchi, Y.; Nishiyama, T.; Kamachi, M. Complex formation of poly(e-caprolactone) with cyclodextrin. Macromol. Rapid Commun. 1997, 18, 535-539. [CrossRef]

23. Iguchi, H.; Uchida, S.; Koyama, Y.; Takata, T. Polyester-containing $\alpha$-cyclodextrin-based polyrotaxane: Synthesis by living ring-opening polymerization, polypseudorotaxanation, and end capping using nitrile N-oxide. ACS Macro Lett. 2013, 2, 527-530. [CrossRef]

24. Dong, T.; He, Y.; Zhu, B.; Shin, K.-M.; Inoue, Y. Nucleation mechanism of $\alpha$-cyclodextrin-enhanced crystallization of some semicrystalline aliphatic polymers. Macromolecules 2005, 38, 7736-7744. [CrossRef]

25. Narayanan, G.; Gupta, B.S.; Tonelli, A.E. Enhanced mechanical properties of poly(E-caprolactone) nanofibers produced by the addition of non-stoichiometric inclusion complexes of poly( $\varepsilon$-caprolactone) and $\alpha$-cyclodextrin. Polymer 2015, 76, 321-330. [CrossRef]

26. Avrami, M. Kinetics of phase change. II transformation-time relations for random distribution of nuclei. J. Chem. Phys. 1940, 8, 212-224. [CrossRef]

27. Avrami, M. Granulation, phase change, and microstructure kinetics of phase change. III. J. Chem. Phys. 1941, 9, 177-184. [CrossRef]

28. Lugito, G.; Woo, E.M. Interior lamellar assembly in correlation to top-surface banding in crystallized poly(ethylene adipate). Cryst. Growth Des. 2014, 14, 4929-4936. [CrossRef]

29. Wu, H.; Qiu, Z. A comparative study of crystallization, melting behavior, and morphology of biodegradable poly(ethylene adipate) and poly(ethylene adipate-co-5 mol \% ethylene succinate). Ind. Eng. Chem. Res. 2012, 51, 13323-13328. [CrossRef]

30. Yang, J.; Pan, P.; Dong, T.; Inoue, Y. Crystallization kinetics and crystalline structure of biodegradable Poly(ethylene adipate). Polymer 2010, 51, 807-815. [CrossRef]

Publisher's Note: MDPI stays neutral with regard to jurisdictional claims in published maps and institutional affiliations.

(C) 2020 by the authors. Licensee MDPI, Basel, Switzerland. This article is an open access article distributed under the terms and conditions of the Creative Commons Attribution (CC BY) license (http://creativecommons.org/licenses/by/4.0/). 